\section{SIMPLIFIED EQUATION TO PREDICT MINIMUM WETTING RATE}

\section{Dear Sir:}

In a previous paper $^{2}$, a correlating equation to predict the minimum wetting rate in a wetted-wall column was proposed as follows:

$$
K y^{2 / 3}+B y^{5 / 3}=A x^{1 / 3}
$$

where $y=\Gamma / \mu, x=\rho \sigma^{3} / \mu^{4} g, B=2 \cdot 3^{5 / 3} / 15$ and $A$ and $K$ are numerical constants evaluated experimentally, which vary depending on the wetted-wall surface properties and liquid distribution manner. Eq. (1) can be used in a wide range of $x$, i. e. $10^{-2}<x<10^{11}$, but the prediction of minimum wetting rate, $\Gamma$, with Eq. (1) contains a trial and error procedure. So it is convenient for practical use to transform Eq. (1) to an explicit function with respect to $y$.

At both extremes Eq. (1) has the asymptotic equations respectively as follows:

$$
\begin{array}{ll}
y=(A / K)^{3 / 2} x^{1 / 2} & \text { for } x \rightarrow 0 \\
y=(A / B)^{3 / 5} x^{1 / 5} & \text { for } x \rightarrow \infty
\end{array}
$$

According to the procedure proposed by Churchill and Usagi ${ }^{11}$, under these conditions the expression

$$
y=\left[\left\{(A / K)^{3 / 2} x^{1 / 2}\right\}^{-n}+\left\{(A / B)^{3 / 5} x^{1 / 5}\right\}^{-n}\right]^{-1 / n}
$$

is suitable for approximation.

Rearranging the above equation, we obtain:

$$
Y=\left[1+Z^{n}\right]^{1 / n}
$$

where

$$
Y=(A / B)^{3 / 5} x^{1 / 5} / y
$$

and

$$
Z=(A / B)^{3 / 5} /\left\{(A / K)^{3 / 2} x^{3 / 10}\right\}
$$

The exponent $n$ in Eq. (2) can be, in this case, evaluated analytically by the following procedure. When $Z=1$, i. e. when $x=(A / B)^{2} /(A / K)^{5}, n=\ln 2 /$ $\ln Y_{Z=1}$.

Substitution of the value of $x$ at $Z=1$ to Eq. (4) gives $Y_{Z=1}=K /\left(B y_{Z=1}\right)$ and $y_{Z=1}$ can be obtained by substituting $x$ at $Z=1$ to Eq. (1) as follows:

$$
\begin{aligned}
K y_{Z=1}{ }^{2 / 3}+B y_{Z=1}{ }^{5 / 3} & =A(A / B)^{2 / 3} /(A / K)^{5 / 3} \\
& =K^{5 / 3} / B^{2 / 3}
\end{aligned}
$$

When $y_{Z=1}=C K / B$, Eq. (6) reduces to

$$
C^{2 / 3}+C^{5 / 3}=1
$$

and this equation gives $C=0.529$.

Hence

$$
Y_{Z=1}=1 / C
$$

and then

$$
n=\ln 2 / \ln (1 / C)=1.09
$$

The above description shows that the exponent $n$

\begin{tabular}{|c|c|c|}
\hline \multicolumn{3}{|c|}{ Nomenclature } \\
\hline & $=$ numerical constant & {$[-$} \\
\hline$g$ & $=$ acceleration due to gravity & {$[\mathrm{cm} / \mathrm{sec}$} \\
\hline$n$ & $=$ exponent defined in Eq. (2) & {$[-$} \\
\hline$x$ & $=\rho \sigma^{3} / \mu^{4} g$ & \\
\hline$Y$ & $=(A / B)^{3 / 5} x^{1 / 5} / y$ & 1 \\
\hline$y$ & $=\Gamma / \mu$ & \\
\hline$Z$ & $=(A / B)^{3 / 5} /\left\{(A / K)^{3 / 2} x^{3 / 10}\right\}$ & \\
\hline$\Gamma$ & $=$ minimum wetting rate & {$[\mathrm{g} / \mathrm{cm} \cdot \mathrm{se}$} \\
\hline$\mu$ & $=$ viscosity of liquid & {$[\mathrm{g} / \mathrm{cm} \cdot \mathrm{se}$} \\
\hline$\rho$ & $=$ density of liquid & {$[\mathrm{g} / \mathrm{cm}$} \\
\hline$\sigma$ & $=$ surface tension of liquid & [dyne/cn \\
\hline
\end{tabular}
in Eq. (2) can be decided independently of $A$ and $K$ so long as it is evaluated by use of the values of $x$ and $y$ at $Z=1$ where $x$ has the value of the intersection of the two asymptotic equations.

When the values of $A$ and $K$ evaluated experimentally in the previous work are used, the equation of the following final form

$$
y=\left[\left\{(A / K)^{3 / 2} x^{1 / 2}\right\}^{-1.09}+\left\{(A / B)^{3 / 5} x^{1 / 5}\right\}^{-1.09}\right]^{-1 / 1.09}
$$

can be used to predict a minimum wetting rate, the deviation from Eq. (1) being less than $6.6 \%$ over the entire range.

\section{Literature Cited}

1) Churchill, S. W. and R. Usagi: AIChE J., 18, 1121 (1972).

2) Munakata, T., K. Watanabe and K. Miyashita: J. Chem. Eng. Japan, 8, 440 (1975).

KAZUNORI WATANABE AND TSUYOSHI MUNAKATA*

Department of Chemical Engineering, Kyushu University, Fukuoka, 812

Received August 11, 1975. 